\title{
Bemerkung zu dem Aufsatze von Herrn Poenisch über den Cometen 1877 III.
}

\section{Von Herrn Dr. Deichmiuller, Observator der Sternwarte zu Bonn.}

Herr Poenisch hat in seiner Bahnbestimmung des Cometen 1877 III (A. N. 2380) eine Bonner Beobachtung dieses Himmelskörpers wegen zu starker Abweichung $\left(+27^{\circ} \mathrm{Ol}\right.$ und $\left.+195^{\prime \prime} 85\right)$ ausgeschlossen. Ich habe daher diese von Dr. Seeliger angestellte Beobachtung nachgesehen, und gefunden, dass kein Rechenfehler vorliegt. Auch die Hypothese, es möchte ein anderer Vergleichstern benutzt sein, fällt weg, da sich kein anderer Vergleichstern von entsprechender Helligkeit in der Nähe befindet. Die Abweichung in AR. legte nun die Vermuthung nahe, dass die Beobachtungszeit um I Stunde falsch angegeben sei, wodurch diese Coordinate mit der Ephemeride innerhalb der Grenzen der plausibeln Beobachtungsfehler in Einklang gebracht wurde, Diese Annahme wurde auch durch den Umstand bestätigt, dass Bonn I88 I, Juni 26. die von Seeliger und mir an jenem Abend beobachtete Zone in die für die Cometenbeobachtung angegebene Zeit hineinreicht. Da ich eine ausreichende Ephemeride für jene Zeit nicht finden konnte, leitete ich aus den beiden noch vorhandenen Beobachtungen dieses Cometen von demselben Abend, nämlich aus Mailand und Odessa seine stündliche Bewegung $7 u+25^{8} 338$ und $+9 \mathrm{I}^{\prime \prime} 2$ ab. Während also hiernach die AR. bis auf $+I^{\mathrm{s}} 67$ der Ephemeride entspricht, bleibt für die Declination 104 "6 6 als Differenz. Ich habe nun mit der obigen Eigenbewegung die Declination auf die Mailänder Bestimmung reducirt, und finde gegen die Ephemeride nur noch eine Abweichung von - $9^{\prime \prime} 2$; es scheint also bei Herrn Poenisch die Vergleichung der beobachteten Declination mit der Ephemeride fehlerhaft zu sein.

\section{Fr. Deichmiiller.}

\section{Beobachtungen des Cometen $a 1881$ (Swift) auf der Leipziger Sternwarte.}

\begin{tabular}{|c|c|c|c|c|c|c|c|c|}
\hline I $88 \mathrm{t}$ & M. $Z$. Leipzig & Are & $A \delta$ & $\begin{array}{l}\text { Von Herrn Dr. Peter. } \\
\text { Vergl. \& app. }\end{array}$ & 1.f.p. & $\delta$ app. & 1.f.p. & Beob. Steris \\
\hline Mai Io & $14^{\mathrm{h}} 24^{\mathrm{m}} 36^{\mathrm{s}}$ & $-1^{m} 48^{\mathrm{s}} 25$ & $+3^{\prime} 6^{\circ} 8$ & $\mathrm{o}^{\mathrm{h}} 38^{\mathrm{m}} 49^{\mathrm{s}} 28$ & $\begin{array}{l}9.593 n \\
0.598 n\end{array}$ & $\begin{array}{r}+26^{\circ} 44^{\prime} 57^{\prime \prime} 4 \\
+2529\end{array}$ & $\begin{array}{l}0.831 \\
0.832\end{array}$ & $\begin{array}{l}\text { Peter } \\
\text { Peter }\end{array}$ \\
\hline
\end{tabular}

Mittlere Oerter der Vergleichsterne für I88I.O $\alpha$ (Wolfers) Red. aufWolfers $\delta$ (Auwers) Red. auf. Auwers Gewicht

$\begin{array}{lllrrr}a & 0^{\mathrm{h}} 40^{\mathrm{m}} 36^{\mathrm{s}} 41 & - & +26^{\circ} 39^{\prime} 48^{\prime \prime 2} & - & - \\ b & 044^{1} 23.03 & +0^{\mathrm{s}} \mathrm{O} & +253826.5 & +0^{\prime \prime} 5 & \text { I } \\ 23.02 & +0.06 & 26.6 & +0.0 & \text { I }\end{array}$

Anschluss an Rümker I I 80 . Weisse II IOZI. Rümker II 307.

Herr Adjunct Palisa war so freundlich den Stern $a$ anzuschliessen. Weisse II ro3 I wurde um ro corrigirt.

Die angewandte Vergrösserung war I 44 fach und erschien der Comet als rundlicher, schwacher, etwas verdichteter Nebel. $\mathbb{C}$ und Dämmerung erschwerten die Beobachtung.

Elements of Comet $b$ 1881. By Professor E. Frisby.

(Communicated by Rear Admiral Fohn Rodgers, Superintendent).

$T=$ i 881 June 16.37001 Wash. m.t.

$\pi=265^{\circ} 31^{\prime} \mathrm{I} 5^{\prime \prime} 4$

$\Omega=270 \quad 5827.0\}$ i 881.0

$i=632555.7$

$\log q=9.866748$

These elements have been computed from the observation made at the Harvard College Observatory June 23, and from two made at Washington June 29 and July 5. The middle place is represented as follows:

$$
\text { (C-O.) } \begin{aligned}
\cos \beta d \lambda & =-13^{\prime \prime} 4 \\
d \beta & =+62.1
\end{aligned}
$$

Equatorial coordinates.

$x=[9.650808] . r \sin \left(v+356^{\circ} 43^{\prime} 26^{\prime \prime} 5\right)$
$y=[9.991846] . r \sin (v+2434252.6)$
$z=[9.961254] . r \sin (v+3284518.1)$
Naval Observatory, Washington $188 \mathrm{I}$, July $\mathrm{I} 6$

\section{In halt:}

Zu Nr. 2387. A. Marth. Ephemerides of the five inner Satellites of Saturn (continued) and of the outer Satellite Japetus I88 $\mathbf{1}$ I882. I6I. E. Weiss. Ueber die telegraphische Mittheilung von Entdeckungen und Beobachtungen. 103. - Th. $\%$. Oppolzer. Praecessions- und Nutationscoefficienten. 165. - Fr. Deichmüller. Ueber den grossen Cometen 6 188ı. 17r. - E. Schönfeld. Antwort auf die Anfrage in Nr. 2384. I7 I. - Fohn Tebbutt. Observations of a Southern Comet $b$ r881. 17r. - Dr. M. Wilhelm Mcyer. Elemente und Ephemeride des Cometen 6188 1. 173. - C. Schrader. Berichtigung der Beobachtung des Cometen $c 1881$ in Nr. 2384 , S. 127, vom 18. Juli. 173. - Fr. Deichmüller. Bemerkung zu dem Aufsatze von Herm Poenisch ïber den Cometen 1877 II I. 175. - Dr. Peter. Beobachtungen des Cometen $a 1881$ (Swift) auf der Leipziger Sternwarte. 175. - - E. Frisby. Elements of Comet $b \mathrm{r} 88 \mathrm{r}$. 